\title{
Effective one-body dynamics in multiple-quantum NMR experiments
}

\author{
E. Rufeil-Fiori, ${ }^{1}$ C. M. Sánchez, ${ }^{1}$ F. Y. Oliva, ${ }^{2}$ H. M. Pastawski, ${ }^{1}$ and P. R. Levstein ${ }^{1, *}$ \\ ${ }^{1}$ Facultad de Matemática, Astronomía y Física and Instituto de Física (CONICET), Universidad Nacional de Córdoba, Ciudad \\ Universitaria, 5000 Córdoba, Argentina \\ ${ }^{2}$ Departamento de Fisicoquímica-INFIQC, Facultad de Ciencias Químicas, Universidad Nacional de Córdoba, Ciudad Universitaria, \\ 5000 Córdoba, Argentina
}

(Received 17 September 2008; revised manuscript received 13 November 2008; published 19 March 2009)

\begin{abstract}
A suitable NMR experiment in a one-dimensional dipolar coupled spin system allows one to reduce the natural many-body dynamics into effective one-body dynamics. We verify this in a polycrystalline sample of hydroxyapatite (HAp) by monitoring the excitation of NMR many-body superposition states: the multiplequantum coherences. The observed effective one-dimensionality of HAp relies on the quasi-one-dimensional structure of the dipolar coupled network that, as we show here, is dynamically enhanced by the quantum Zeno effect. Decoherence is also probed through a Loschmidt echo experiment, where the time reversal is implemented on the double-quantum Hamiltonian, $\mathcal{H}_{D Q} \propto I_{i}^{+} I_{j}^{+}+I_{i}^{-} I_{j}^{-}$. We contrast the decoherence of adamantane, a standard three-dimensional system, with that of HAp. While the first shows an abrupt Fermi-type decay, HAp presents a smooth exponential law.
\end{abstract}

DOI: 10.1103/PhysRevA.79.032324 PACS number(s): 03.67.Pp, 03.65.Xp, 76.60.Lz, 76.90.+d

\section{INTRODUCTION}

The new developments in nanodevices [1,2], spintronics [3], and quantum information processing [4] critically rely on the control of quantum dynamics. This control is challenging because the manipulation of quantum states [5] is crucially limited by decoherence [6,7]. In this sense, much can be learned from nuclear magnetic resonance [8,9], which offers the opportunity to tailor the interactions, and thus the time scales, and to quantify decoherence by implementing Loschmidt echoes [10].

The control of interaction anisotropy, e.g., the switch from a dipolar to an $X Y$ (planar) interaction, provides a tool for enhancing the transfer of quantum information $[11,12]$. In particular, the interactions can be sequentially turned on and off to prune some branches in real space so that an excitation is directed to a desired target through a specific pathway [13]. By exploiting the mapping between spins and fermions, spin state transfer in linear spin chains and rings coupled by $X Y$ interaction was proposed [14] and observed in liquidstate NMR [15]. Moreover, new suggestions that improve state transfer have been reported [16-18]. The structurally quasi-one-dimensional spin systems of hydroxyapatite (Hap) and fluorapatite have been proposed as candidates for implementing quantum information processing in solid-state NMR [19]. In these systems, universal control has been achieved by implementing collective control together with suitable spin manipulation at the chain ends [20,21]. All these fine control attempts might be frustrated by decoherence [22-24]. Thus, the dependence of decoherence on nuclear-spin network topology becomes an important issue.

In this work, we tailor the interactions in a onedimensional (1D) dipolar coupled spin system to transform its natural many-body dynamics into effective one-body dynamics. The difference in dynamics is observed through the

\footnotetext{
*patricia@famaf.unc.edu.ar
}

excitation of NMR many-body superposition states: the multiple-quantum coherences [25]. Each $M$-quantum coherence $(M-\mathrm{QC})$ collects all the superpositions between two Zeeman states whose difference in total magnetic moment is the integer $M . M$-QC intensities are tested in solid-state NMR through phase codification techniques that allow one to follow the superposition weights as they are being created [26].

In a homogeneous one-dimensional chain of nuclear spin $1 / 2$, all spin sites have the same energy and couplings. If the spins are coupled under double-quantum interactions, $\mathcal{H}_{D Q}$ $\propto I_{i}^{+} I_{j}^{+}+I_{i}^{-} I_{j}^{-}$, restricted to nearest neighbors (NNs), analytical methods give closed expressions for the intensities of the multiple-quantum coherences [27]. Although $\mathcal{H}_{D Q}$ acting on a thermal equilibrium state excites all even-order coherences, it can be proved that in a one-dimensional system only zeroand second-order coherences are allowed [27]. The results of this model are compared with numerical calculations that include more realistic interactions and with NMR experiments in a polcrystalline sample of HAp. HAp behaves as a quasi-one-dimensional spin chain due to its dipolar coupled network structure $[28,29]$. We show that this anisotropy is further enhanced by a dynamical quantum Zeno effect (QZE).

Decoherence is tested experimentally in HAp through a Loschmidt echo variant [10] based on $\mathcal{H}_{D Q}$ and its reversal. The same experiment is performed in adamantane, a typical three-dimensional (3D) system, allowing us to contrast the effect of the coupling network.

This paper is organized as follows. Section II discusses the multiple-quantum coherences as well as the doublequantum Hamiltonian. Here, the theoretical basis that allows one to obtain the effective one-body dynamics is summarized. Section III describes the crystallographic and dynamical properties of HAp which make it an effective onedimensional system. Section IV describes the experimental methods. Sections V and VI respectively present numerical and experimental results for the $M$-QC dynamics. Section VII is devoted to the conclusions. 


\section{MULTIPLE-QUANTUM COHERENCE AND EFFECTIVE ONE-BODY DYNAMICS}

In a typical solid-state NMR experiment on a system of $N$ identical spins $1 / 2$, the main interaction can be described by a dipolar Hamiltonian truncated with respect to the dominant Zeeman interaction [30]:

$$
\begin{aligned}
\mathcal{H}_{\text {ZZ }} & =\sum_{i, j} \frac{d_{i j}}{2}\left(2 I_{i}^{z} I_{j}^{z}-I_{i}^{x} I_{j}^{x}-I_{i}^{y} I_{j}^{y}\right) \\
& =\sum_{i, j} \frac{d_{i j}}{2}\left(2 I_{i}^{z} I_{j}^{z}-\frac{I_{i}^{+} I_{j}+I_{i}^{-} I_{j}^{+}}{2}\right),
\end{aligned}
$$

where $d_{i j}=\left[\gamma^{2} \hbar^{2} /\left(2 r_{i j}^{3}\right)\right]\left[3 \cos ^{2}\left(\theta_{i j}\right)-1\right]$ are the dipolar couplings, with $\theta_{i j}$ as the angle between the internuclear vector $\mathbf{r}_{i j}$ and the external magnetic field, and $\gamma$ as the gyromagnetic ratio. $I_{i}^{z}$ are the $z$ components of the spin operators defined by the direction of the static magnetic field, and $I_{i}^{+}$and $I_{i}^{-}$are the raising and lowering operators. In dipolar coupled spin systems at high magnetic field, the off-diagonal elements of the density matrix in the $z$ basis, i.e., the coherences $\rho_{r s}$ $=\langle r|\rho| s\rangle$, can be labeled by the difference in the total magnetic quantum numbers between the states involved in the transition, $M=m_{r}-m_{s}$, where $I^{z}|s\rangle=m_{s}|s\rangle$, with $I^{z}=\sum_{i} I_{i}^{z}$. All the elements of the density matrix that connect two states whose difference in total magnetic moment is $M$ contribute to the intensity of an $M$-QC [24]. Although only singlequantum coherences $(M= \pm 1)$ are directly observed by NMR, phase codification techniques [25] allow one to obtain information on the multiple-quantum coherences.

In order to create coherences from an initial thermal equilibrium state, a Hamiltonian which does not commute with its density matrix is necessary. Both the dipolar Hamiltonian rotated to the $x$ axis $\left(\mathcal{H}_{X X}\right)$ and the double-quantum Hamiltonian $\left(\mathcal{H}_{D Q}\right)$ fulfill this requirement and are experimentally achievable:

$$
\begin{gathered}
\mathcal{H}_{X X}=\exp \left(-i \frac{\pi}{2} I^{y}\right) \mathcal{H}_{Z Z} \exp \left(i \frac{\pi}{2} I^{y}\right) \\
=\sum_{i, j} \frac{d_{i j}}{2}\left(2 I_{i}^{x} I_{j}^{x}-I_{i}^{y} I_{j}^{y}-I_{i}^{z} I_{j}^{z}\right), \\
\mathcal{H}_{D Q}=\sum_{i, j} \frac{d_{i j}}{2}\left(I_{i}^{x} I_{j}^{x}-I_{i}^{y} I_{j}^{y}\right) \\
=\sum_{i, j} \frac{d_{i j}}{4}\left(I_{i}^{+} I_{j}^{+}+I_{i}^{-} I_{j}^{-}\right) .
\end{gathered}
$$

In the special case of nearest neighbor (NN) interactions, $\mathcal{H}_{D Q}$ is unitary similar to the $X Y$ Hamiltonian, $\mathcal{H}_{X Y} \propto I_{i}^{+} I_{j}^{-}$ $+I_{i}^{-} I_{j}^{+}$. Consequently, $\mathcal{H}_{D Q}$ can simulate the $\mathcal{H}_{X Y}$ dynamics after the corresponding transformation of the initial state. Although this relation between $\mathcal{H}_{D Q}$ and $\mathcal{H}_{X Y}$ is valid in one, two, and three dimensions [20,31], we focus on onedimensional systems, for which closed analytical results are available. Here, we summarize the successive transforma-

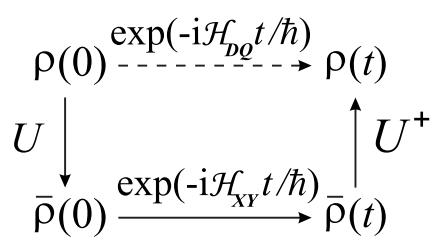

FIG. 1. Pathways to generate multiple-quantum coherences from the initial state $\rho(0)$. Experimentally one follows the dashed arrow. This is equivalent, in a 1D system with nearest-neighbor interactions, to the mathematical pathway indicated in solid arrows.

tions, developed by Doronin et al. [27], that enable this mapping. First, one applies the unitary transformation

$$
U=\exp \left(-i \pi I_{2}^{x}\right) \exp \left(-i \pi I_{4}^{x}\right) \cdots \exp \left(-i \pi I_{2 n}^{x}\right) \cdots
$$

to $\mathcal{H}_{D Q}$. This is a composition of $\pi$ pulses which rotate evennumbered spins $180^{\circ}$ about the $x$ axis. As a result, the transformed Hamiltonian is

$$
\mathcal{H}_{X Y}=U \mathcal{H}_{D Q} U^{\dagger}=\sum_{i} \frac{d_{i, i+1}}{4}\left(I_{i}^{+} I_{i+1}^{-}+I_{i}^{-} I_{i+1}^{+}\right) .
$$

The same transformation must be applied to the initial state. For the thermal equilibrium state, in the high-field and hightemperature limit, we only consider the main deviation of the density matrix from the identity, which is the experimentally observable part, i.e., $\rho(0)=\sum_{i} I_{i}^{z}$. This leads to

$$
\bar{\rho}(0)=U \rho(0) U^{\dagger}=\sum_{i}(-1)^{i-1} I_{i}^{z} .
$$

Then, as shown schematically in Fig. 1, the dynamics of an initial state $\rho(0)$ under $\mathcal{H}_{D Q}$ is reduced to the dynamics of $\bar{\rho}(0)$ under $\mathcal{H}_{X Y}$ which, in turn, maps to a noninteracting fermion system [32-34]. The dynamics of this fermionic system has a closed analytical solution when the interaction is homogeneous, $d_{i, i+1}=d, \forall i$. Transforming back to the double-quantum dynamics, a closed expression for the density matrix $\rho(t)$ can be obtained. The intensities $J_{M}$ of the $M-\mathrm{QC}$ are calculated as

$$
J_{M}(t)=\operatorname{Tr}\left\{\rho_{M}(t) \rho_{-M}(t)\right\},
$$

where

$$
\rho_{M}(t)=\sum_{r, s}^{\prime} \rho_{r s}(t)
$$

where $\Sigma^{\prime}$ restricts the sum to $m_{r}-m_{s}=M$. Thus, $\rho_{M}$ collects all the contributions to $\rho$ due to coherences of order $M$, and $\rho(t)=\Sigma_{M} \rho_{M}(t)$. Then, the $J_{M}(t)$, in the normalized form $\Sigma_{M} J_{ \pm M}=1$, result in

$$
\begin{gathered}
J_{0}(t)=\frac{1}{N} \sum_{n} \cos ^{2}\left[4 d t / \hbar \cos \left(\frac{\pi n}{N+1}\right)\right], \\
J_{ \pm 2}(t)=\frac{1}{2 N} \sum_{n} \sin ^{2}\left[4 d t / \hbar \cos \left(\frac{\pi n}{N+1}\right)\right],
\end{gathered}
$$

with $n=1, \ldots, N$. This shows that only Z-QC and 2-QC are allowed. All other even orders cannot be created. Even 
though a closed analytical solution is not possible in a NN inhomogeneous case, it was shown that only zero- and second-order coherences are excited [35], as what occurs in the homogeneous chain.

Finally, the evolution of particular initial conditions [20] under 1D nearest-neighbor double-quantum interactions reduces to that of noninteracting ("one-body") spinless fermions. This one-body dynamics manifests through the presence of only 2 orders of coherence (Z-QC and 2-QC). We test this in Secs. V and VI by performing numerical simulations and multiple-quantum NMR experiments of the dynamics under $\mathcal{H}_{D Q}$. This is contrasted with the irreducible many-body dipolar dynamics under $\mathcal{H}_{X X}$.

\section{DYNAMICAL ENHANCEMENT OF THE ONE- DIMENSIONALITY BY THE QUANTUM ZENO EFFECT}

We perform NMR experiments in a physical system that behaves as a one-dimensional spin- $1 / 2$ chain. The system is a polycrystalline sample of hexagonal hydroxyapatite, $\mathrm{Ca}_{5}\left(\mathrm{PO}_{4}\right)_{3} \mathrm{OH}$, with space group $P 63 / \mathrm{m}$. Due to the difference in resonance frequencies of the various spin nuclei, the experimental setup allows taking account of only the spin degrees of freedom of the ${ }^{1} \mathrm{H}$ nuclei. The hydrogen spins of this sample are ordered as linear chains in the $c$ direction of a hexagonal arrangement $(a=b, c)$ [29]. A central chain is surrounded by six neighboring chains at a distance of $r_{x}$ $=9.42 \AA \quad\left(r_{x}=a\right)$. The closest distance between protons within a chain is $r_{\text {in }}=3.44 \AA\left(r_{\text {in }}=c / 2\right)$. In solid-state NMR the strongest interaction is the dipolar one. Because of the dependence of the dipolar couplings on the spin distance, the ratio between the in-chain $\left(d_{\text {in }}\right)$ and the cross-chain $\left(d_{x}\right)$ dipolar couplings for the orientation that maximizes the inchain coupling is

$$
\frac{d_{\text {in }}}{d_{x}}=2\left(\frac{r_{x}}{r_{\text {in }}}\right)^{3} \approx 2 \times 20 .
$$

As we work with a polycrystal, we calculate for each chain orientation the ratio of the local second moment due to inchain interactions, $M_{2 \text {,in }}$, to the local second moment due to the six neighboring chains, $M_{2, x}$. Then, by taking the average over solid angle, we obtain

$$
\sqrt{\left\langle\frac{M_{2, \text { in }}}{M_{2, x}}\right\rangle}=\langle f(\theta, \phi)\rangle\left(\frac{r_{x}}{r_{\text {in }}}\right)^{3} \approx 1.5 \times 20,
$$

where $f(\theta, \phi)$ is the angular function that takes into account the angular dependence of the dipolar interaction and the relative orientation of the internuclear vectors with respect to the external magnetic field.

There is a dynamical effect that further enhances the difference between these two couplings. The characteristic time for a flip-flop process within the chain is clearly

$$
\tau_{\text {in }} \approx \frac{\hbar}{d_{\text {in }}} .
$$

However, the characteristic rate of a flip-flop due to the weak cross-chain couplings should be estimated invoking the Fermi golden rule that yields [36]

$$
\frac{1}{\tau_{x}} \approx \frac{1}{\hbar} d_{x}^{2} \frac{1}{d_{\text {in }}},
$$

and not $d_{x} / \hbar$ as one might first guess. This is because the strong in-chain dynamics leads to an uncertainty of the final state over a wide excitation spectrum. Then, we have

$$
\frac{\tau_{\text {in }}}{\tau_{\mathrm{x}}} \approx\left(\frac{d_{x}}{d_{\text {in }}}\right)^{2} \approx\left(\frac{r_{\text {in }}}{r_{x}}\right)^{6} \approx \frac{1}{400} .
$$

Equation (17) states that fast in-chain dynamics makes already slow cross-chain dynamics even slower. This is a form of the QZE, which states that quantum dynamics is slowed down by a frequent measurement process [37]. Spindiffusion experiments in low-dimensional crystals showed an unexpected dimensional crossover as a function of a structural parameter [38]. This crossover was described as a QZE where the internal degrees of freedom act as measurement apparatus [39]. The concept that the measurement is played by an interaction with another quantum object, or simply another degree of freedom of the subsystem investigated, was independently and fully formalized by recasting it in terms of an adiabatic theorem in Ref. [40]. It can even lead to a freeze of the spin swap dynamics as observed in a crosspolarization experiment [11]. In the present context, Eq. (18) reinforces the effective one-dimensional behavior of HAp.

\section{NMR EXPERIMENTAL SETUP}

The experiments were performed using a Bruker Avance II spectrometer operating at a ${ }^{1} \mathrm{H}$ resonance frequency of 300.13 MHz. We used a cross polarization-magic angle spinning (CP-MAS) probe working in static conditions at room temperature with a $4 \mathrm{~mm}$ outer diameter rotor.

The characterization of the dynamics of the multiplequantum coherences was performed using the pulse sequences shown in Fig. 2. The different orders of coherence excited under $\mathcal{H}_{D Q}$ were generated using the two-pulse sequence shown in Fig. 2(a) $[41,42]$. With this sequence, $\mathcal{H}_{D Q}$ is built after a minimum number of scans $N_{s}$, with $N_{s}$ $=2 M_{\text {des }}$, where $M_{\text {des }}$ is the order of coherence one desires to detect indirectly. Thus, in order to measure 2-QC, a minimum of four scans must be added. To get a better signal-tonoise ratio, the total number of scans must be a multiple of $N_{s}$. Therefore, the evolution of $M_{\mathrm{des}}$-QC under $\mathcal{H}_{D Q}$ is built after $N_{s}$ scans by adding signals with different phases $\phi$. In particular, one uses $\phi=0, \pi / 2, \pi$, and $3 \pi / 2$ for filtering the 2 -QC and $\phi=0, \pi / 4, \pi / 2,3 \pi / 4, \pi, 5 \pi / 4,3 \pi / 2$, and $7 \pi / 4$ for filtering the 4-QC. In both cases, the phase of the reading pulse was alternated between 0 and $\pi$ to keep only the orders of coherences $M_{\mathrm{des}} \pm n N_{s}$, with $n=0,1,2, \ldots[26,43]$.

In order to encode $M$-QC orders during the evolution under $\mathcal{H}_{X X}$ [Eq. (4)], we used the sequence shown in Fig. 2(b), which is a modified version of the sequence reported in Ref. [44]. Here, the highest coherence order detected, $n_{\max }=8$, is governed by the phase shift increment $\Delta \phi=\pi / n_{\max }$.

In these sequences, the recorded free induction decays (FIDs) were the sum of 64 scans. The recycling time, $D_{1}$ $=3 \mathrm{~s}$, was chosen to be longer than five times the spin-lattice relaxation time $T_{1} \approx 500 \mathrm{~ms}$. The $\pi / 2$ pulse length was 
(a)

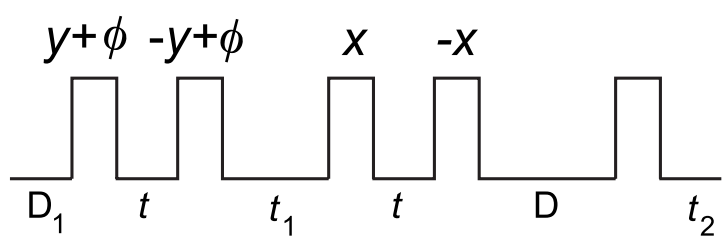

(b)

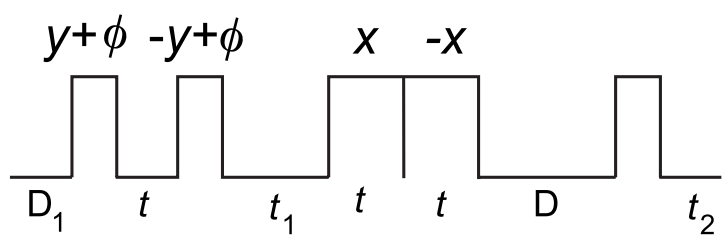

(c)

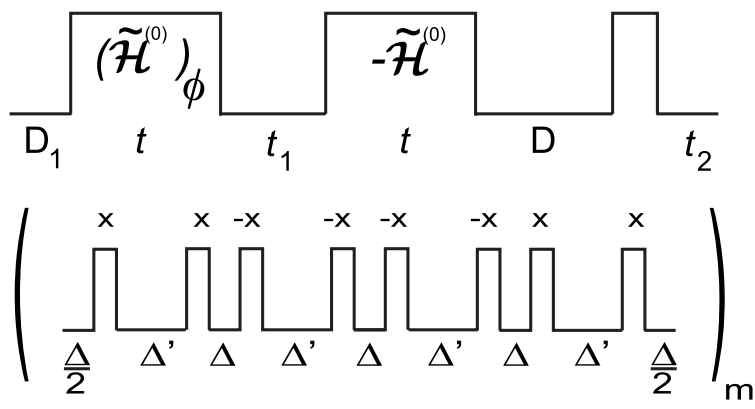

FIG. 2. (a) Selective two-pulse sequence to generate $M$-QC under an average double-quantum Hamiltonian $\mathcal{H}_{D Q}$ based on rotations of $\mathcal{H}_{\mathrm{ZZ}}$ (free evolution). It detects 2 -QC $(2+4 n)$ or $4-\mathrm{QC}(4$ $+8 n$ ) intensities by applying the appropriate phase cyclings (see text). All pulses are of $\pi / 2$. (b) Sequence to generate $M$-QC under a rotated dipolar Hamiltonian $\mathcal{H}_{X X}$. (c) Pulse sequence to generate $M$-QC under a $\mathcal{H}_{D Q}$ based on $m$ repetitions of the eight $\pi / 2$-pulse pattern displayed in parenthesis. In (b) and (c) a free induction decay was recorded for each value of $t$ and $\phi$. The highest coherence order detected, $n_{\max }=8$, is governed by a digital phase shift increment, with $\Delta \phi=\pi / n_{\max }$.

$2.74 \mu$ s. The preparation times $t$, i.e., the periods evolving under the desired effective Hamiltonian, were varied from 1 to $200 \mu \mathrm{s}$. The free evolution time $t_{1}=0.5 \mu \mathrm{s}$ was negligible. After the mixing time and before the $\pi / 2$ reading pulse, a delay $D=2 \mathrm{~ms}$ was used to allow the transverse magnetization to decay. The detected signal was normalized to a reference FID obtained by the application of a $\pi / 2$ pulse with the same number of scans.

The two-pulse sequence used to generate $\mathcal{H}_{D Q}$ was chosen because the fast growth of the 2-QC intensity is not captured with the eight-pulse sequence shown in Fig. 2(c) [25]. The last only captures a few data points in the time range of interest because of the minimum time of $\sim 60 \mu$ s required to accommodate the eight pulses of the basic unit. However, the eight-pulse sequence was applied to implement a "Loschmidt echo" experiment, that is, to generate $\mathcal{H}_{D Q}$ and then $-\mathcal{H}_{D Q}$, by using $\phi=0$. We use this echo to give a measure of decoherence rates. In order to compare this decoherence rate in HAp with a widely known system, we performed the echo experiments in adamantane. Adamantane is a $3 \mathrm{D}$ molecular crystal with only intermolecular dipolar interactions [45] (the intramolecular interactions cancel out due to rapid molecular rotations). In the Loschmidt echo experiments, the preparation time was varied from 60 to $1400 \mu \mathrm{s}$ and the $\pi / 2$ pulse length was $2.34 \mu$ s for HAp and $2.20 \mu$ s for adamantane. The experiments were carried out in a polycrystalline sample of hydroxyapatite synthesized by a modification of the biomimetic method reported by Zhang et al. [46], while a commercial polycrystalline sample of adamantane was used as provided.

\section{NUMERICAL RESULTS: MULTIPLE-QUANTUM DYNAMICS}

The $M$-QC intensities were numerically simulated using an ensemble average of the evolution of each Zeeman state. The total magnetization was calculated as a function of preparation time $t$ and as a function of the $M$-QC codification phase $\phi$. This was obtained by evolving each initial state under $\mathcal{H}$ during $t$ and then under $-\mathcal{H}_{\phi}$, where $\mathcal{H}_{\phi}=\exp ($ $\left.-i \phi I^{z}\right) \mathcal{H} \exp \left(i \phi I^{z}\right)$. Finally, a fast Fourier transform on $\phi$ was applied to the magnetization to obtain the $M$-QC intensities $J_{M}(t)[25]$.

An alternative method to obtain $J_{M}(t)$, which makes use of Eq. (10), was used. In this case, the $\rho_{M}$ are obtained from the elements of the density matrix calculated for each Zeeman state. Although this method is time consuming, it clearly shows how the different coherences contribute to the intensity of a given order.

This second method allows us to draw some conclusions about the unitary transformations schematized in Fig. 1. Even when $\mathcal{H}_{D Q}$ is unitary similar to $\mathcal{H}_{X Y}$, an arbitrary initial condition under $\mathcal{H}_{D Q}$ does not necessarily yield only 2 orders of coherences. In a chain with NN $X Y$ interaction, any excitation remains in the same subspace, i.e., only zero-order coherences appear. However, the transformed initial thermal equilibrium condition $\bar{\rho}(0)$ [Eq. (9)] imposes a further restriction in the accessible Hilbert space in which this condition can evolve under $X Y$ interaction. In this case, only a portion of the ZQ subspace can be reached. It is because of this restriction that, after transforming back to the doublequantum dynamics $\rho(t)$, only zero- and second-order coherences are excited.

In order to obtain the dynamics of $J_{M}(t)$ under $\mathcal{H}_{D Q}$ and contrast this with that under $\mathcal{H}_{X X}$, we used the first method described above. Since the effective Hamiltonians $\mathcal{H}_{D Q}$ and $\mathcal{H}_{X X}$ are built up experimentally from the natural dipolar interaction, which decays with $1 / r^{3}$, it becomes important to take into account the next-nearest-neighbor (NNN) interaction in the simulations. In a chain, the values of the NNN couplings are $1 / 8$ of the $\mathrm{NN}$ ones. The simulated dynamics of the Z-QC, 2-QC, and 4-QC intensities under $\mathcal{H}_{X X}$ and $\mathcal{H}_{D Q}$ is shown in Figs. 3 and 4 for an $N=10$ spin chain starting at thermal equilibrium. Preliminary experimental results in polycrystalline HAp showed that there were no detectable $M$-QC intensities after $200 \mu$ s. Consequently, we do not need simulations for longer times, but we have to take a large enough number of spins to avoid distortions of the dynamics due to reflections at the chain ends. To verify this, we calculated the earliest time at which the mesoscopic echo, i.e., the revival that appears because of the finite nature of the system 


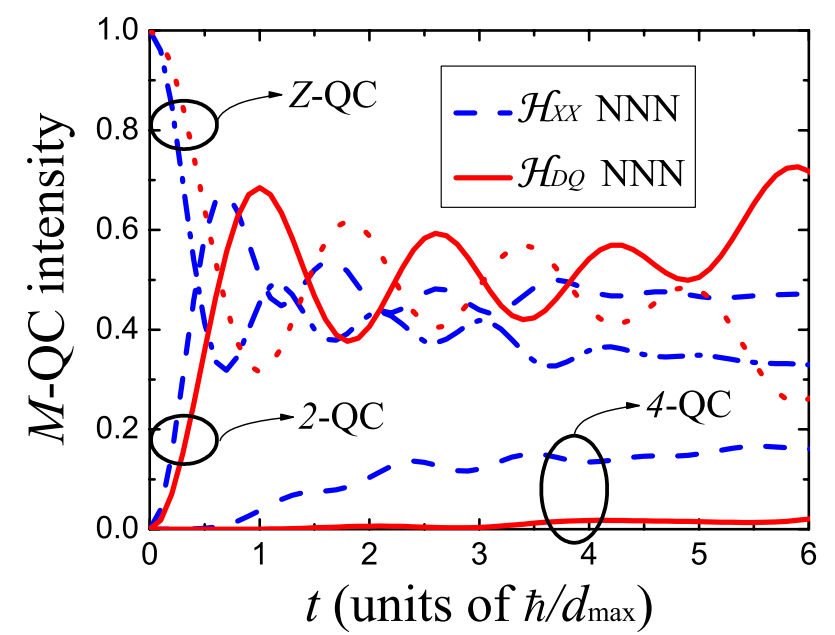

FIG. 3. (Color online) Numerical simulations of the dynamics of the Z-QC, 2-QC, and 4-QC intensities of HAp under $\mathcal{H}_{X X}$ (dashed line for 2-QC and 4-QC, and dash-dotted line for Z-QC) and $\mathcal{H}_{D Q}$ (solid line for 2-QC and 4-QC, and dotted line for Z-QC) in a ten-spin chain with NNN interaction for the chain orientation that maximizes the coupling, $d_{\max } / \hbar=2 \pi \times 2.95 \mathrm{kHz}$. The mesoscopic echo appears at $6 \hbar / d_{\max }$.

$[14,47]$, occurs. This is ensured by using a single crystal at orientation $\theta_{i j}=0$, leading to the maximum dipolar coupling $d_{\text {max }}$, which for HAp is $d_{\max }=2 \pi \hbar \times(2.95 \mathrm{kHz})$. Any other orientation will just stretch the time scale of this curve, delaying the occurrence of the mesoscopic echo. As shown in Fig. 3, for ten spins the mesoscopic echo appears at $6 \hbar / d_{\max } \approx 325 \mu \mathrm{s}$. It is important to emphasize that by varying slightly the number of spins, the dynamics changes only in the neighborhood of the mesoscopic echo, remaining unaffected before $3.7 \hbar / d_{\max } \approx 200 \mu \mathrm{s}$.

In Fig. 4, the 4-QC intensity dynamics in a ten-spin chain of HAp with NNN interactions under $\mathcal{H}_{D Q}$ and $\mathcal{H}_{X X}$ is dis-

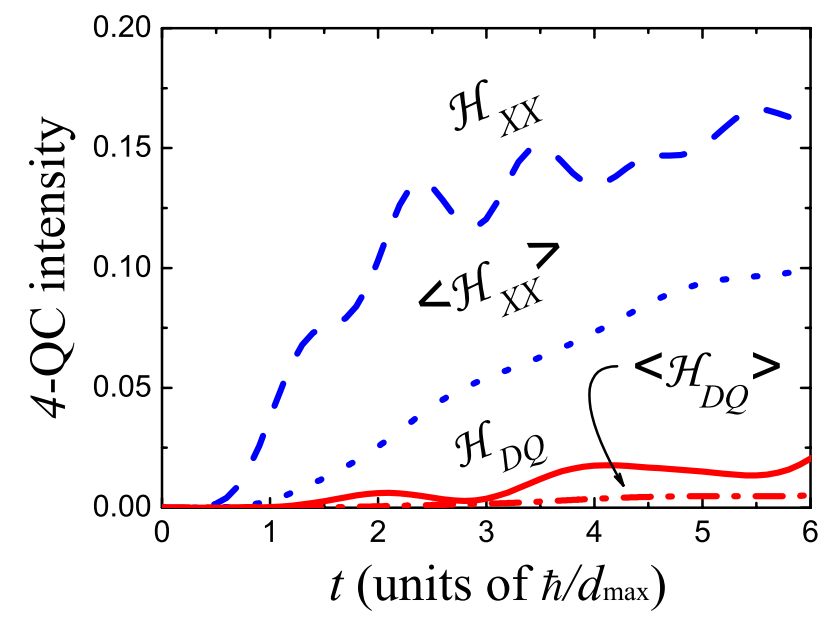

FIG. 4. (Color online) Numerical simulations of the dynamics of the 4-QC intensities under $\mathcal{H}_{X X}$ and $\mathcal{H}_{D Q}$ for a ten-spin chain of HAp with NNN interactions. Dashed line corresponds to $\mathcal{H}_{X X}$ and solid line corresponds to $\mathcal{H}_{D Q}$, both at the orientation of maximum dipolar coupling, $d_{\max } / \hbar=2 \pi \times 2.95 \mathrm{kHz}$. The powder average is shown with a dotted line for $\left\langle\mathcal{H}_{X X}\right\rangle$ and with dash-dotted line for $\left\langle\mathcal{H}_{D Q}\right\rangle$.

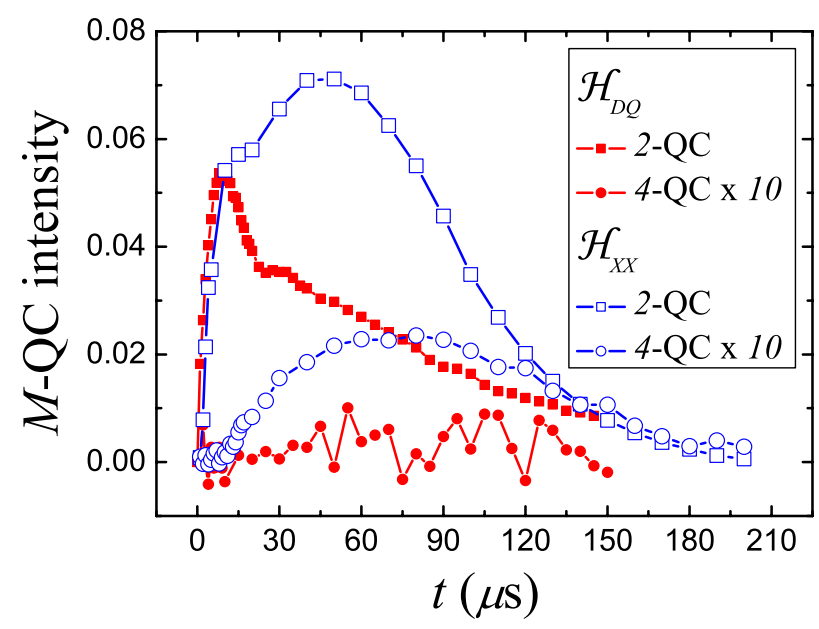

FIG. 5. (Color online) Dynamics of 2-QC and 4-QC intensities under $\mathcal{H}_{D Q}$ and $\mathcal{H}_{X X}$ in HAp implemented with the pulse sequence of Figs. 2(a) and 2(b), respectively. Notice that the normalized intensities of the 4-QC are ten times enlarged.

played. In each Hamiltonian evolution, a single orientation of the chain and a powder average (the integral over solid angle of the orientation dependent dynamics) were calculated. Notice that the observable we are using to check the effective one-body dynamics is robust under orientation average; i.e., the nonexcitability of the 4-QC occurs for every orientation of the chains in a polycrystalline sample, maintaining its null intensity. As can be seen, if one includes the NNN interactions in the chain, the intensity of the 4-QC under $\mathcal{H}_{D Q}$ is not strictly zero. However, this intensity will not be observed under the typical conditions of an NMR experiment. In contrast, the intensity of a 4-QC under $\mathcal{H}_{X X}$ might be observable.

The inclusion of an extra interaction, in this case the NNN interaction, breaks the mapping to noninteracting fermions. Consequently, the system evolution is no longer restricted to only Z-QC and 2-QC. However, as it is clearly shown in Fig. 4 , the 4-QC under $\mathcal{H}_{D O}$ is 1 order of magnitude smaller than the 4-QC excited by $\mathcal{H}_{X X}$. This means that $\mathcal{H}_{D Q}$ still keeps the main dynamics between Z-QC and 2-QC. Hence, one can infer that the effective one-body dynamics is preserved as a good approximation.

\section{EXPERIMENTAL RESULTS: MULTIPLE-QUANTUM DYNAMICS AND DECOHERENCE}

The pulse sequences shown in Figs. 2(a) and 2(b) were used to generate $M$-QC under the effective Hamiltonians $\mathcal{H}_{D Q}$ and $\mathcal{H}_{X X}$, respectively, from a thermal equilibrium state. Figure 5 displays the 2-QC and 4-QC intensities as functions of the preparation time $t$. There, the 4-QC has been multiplied by a factor of 10 because of its small intensity as compared with the 2-QC. While the 4-QC intensity under $\mathcal{H}_{X X}$ is well above the noise level, being evident its growth and decay, the intensity of the $4-\mathrm{QC}$ under $\mathcal{H}_{D Q}$ remains at the noise level.

In our particular $1 \mathrm{D}$ system, the essential difference between the ideal $\mathcal{H}_{X X}$ and $\mathcal{H}_{D Q}$ is that the first allows for the 


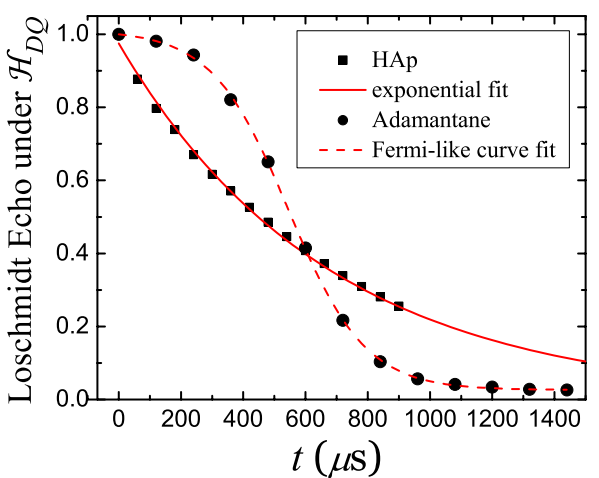

FIG. 6. (Color online) Loschmidt echo experiment based on $\mathcal{H}_{D Q}$ and $-\mathcal{H}_{D Q}$ using the sequence shown in Fig. 2(c) with $\phi=0$ in HAp (squares) and adamantane (circles).

development of many orders of coherence, while the second allows only 2 . Since higher orders of coherences decay at higher rates $[48,49]$, we expect a faster decoherence in the case of $\mathcal{H}_{X X}$. However, we should also assess the precision of the experimental sequences used to generate these Hamiltonians. In this context, one should remember that our implementation of a multiple-quantum experiment under $\mathcal{H}_{X X}$ includes a dipolar Hamiltonian reversal. This involves a further truncation of the dipolar Hamiltonian with respect to the rf Zeeman interaction during the long rf pulse [30]. This produces additional decoherence because the truncated nonsecular terms, whose magnitudes depend on the rf power, are not reversed [23]. The pulse sequences to generate $\mathcal{H}_{D Q}$ may also have some limitations. It is known that the eight-pulse sequence produces a much better average Hamiltonian than the two-pulse one, especially for long preparation times $[25,50]$. This is because the two-pulse sequence plotted in Fig. 2(a) does not average out the chemical shift nor cancels out $\mathrm{rf}$ inhomogeneities as the eight-pulse sequence does. For example, if we compare the 2-QC intensities in HAp for the eight- and two-pulse sequences, both of them show exponential decay. However, the characteristic time of the first is $\tau_{8 \mathrm{p}} \approx 210 \mu$ s, while that of the second is $\tau_{2 \mathrm{p}} \approx 65 \mu$ s; i.e., it is three times faster. The rapid decay of the 2-QC intensity with the two-pulse sequence explains the early occurrence of the maximum $(\approx 15 \mu \mathrm{s})$ in the evolution of 2-QC (see Fig. $5)$ as compared with the theoretical estimation of $\hbar / d_{\max }$ $\approx 50 \mu$ s in Fig. 3. Because of this, the decay of the 2-QC for the two-pulse sequence is not a reliable quantifier of the decoherence of the system.

In order to have a measure of the global decoherence time of the system under $\mathcal{H}_{D Q}$, we used the eight-pulse sequence shown in Fig. 2(c). Having minimized possible experimental artifacts, we expect to have a decoherence that reflects the properties of the sample itself (topology of the coupling network, defects, etc.). Following this idea, we compare the behaviors of HAp and adamantane measuring a Loschmidt echo, that is, generating $\mathcal{H}_{D Q}$ and then $-\mathcal{H}_{D Q}$. The decays of both systems are displayed in Fig. 6. The difference in the functional form of decay is remarkable. While a simple exponential with characteristic time $\tau_{\varphi}=(770 \pm 50) \mu \mathrm{s}$ holds for HAp, a Fermi-type curve $M(t) \propto 1 /\left\{1+\exp \left[\left(t-t_{c}\right) / \tau_{\varphi}\right]\right\}$, with $t_{c}=(545 \pm 2) \mu \mathrm{s}$ and $\tau_{\varphi}=(123 \pm 2) \mu \mathrm{s}$, provides the best fit for adamantane.
It should be noticed that in adamantane, coherences of very high order are generated quite rapidly. Indeed, coherences of orders $M>100$ are well defined after $0.5 \mathrm{~ms}$ [48,51], indicating the huge portion of the Hilbert space explored through $\mathcal{H}_{D Q}$ in this system. As shown by the Fermitype curve, the coherence of such highly interacting system is not sustained beyond a critical time $t_{c}$ where a sort of "catastrophe" seems to occur. A similar behavior is observed in simulations of highly interacting systems, either fermions or bosons, whose coherence also decays following a Fermitype curve $[52,53]$. In those works, a self-consistent approximation allows one to see this critical stage as the triggering of a nonlinear loop.

In contrast with adamantane, the decoherence of HAp, as seen from the Loschmidt echo, occurs smoothly following an exponential law. This sort of decay has been seen in chaotic one-body systems in semiclassical states where the perturbation effects are limited [54-56]. Hence, this decay is consistent with the restricted dynamics imposed by the low connectivity of a 1D system. Furthermore, as the dominant dynamics is that of the noninteracting fermions, the residual interactions and the experimental imperfections define the "environment" that produces the exponential decoherence.

Although the observed decoherence rate seems to be somewhat fast to enable a straightforward quantum information application, the exponential decay of the dynamics of the 1D system may be easier to manipulate than the dynamics of the 3D system. On the other hand, the 3D system presents a short-time behavior that could be nicely exploited to implement quantum operations, because the coherence is lost at a very low rate. Further experimental designs are necessary to confirm the origin of these different functional forms, and to quantify the factors determining the respective characteristic decay times $\tau_{\varphi}$ in HAp and $t_{c}$ and $\tau_{\varphi}$ in adamantane.

\section{CONCLUSION}

We have shown that the $M$-QC intensities under a doublequantum Hamiltonian in HAp behave as effective one-body dynamics. This has been observed through several experiments where the evolutions of the intensities of the 2-QC and 4-QC were studied under the action of $\mathcal{H}_{D Q}$. These results were contrasted with the many-body dynamics induced by $\mathcal{H}_{X X}$. No coherence orders above 2 appear under $\mathcal{H}_{D Q}$, while they do appear under $\mathcal{H}_{X X}$. In both cases, the dynamics remains mainly one dimensional as the natural anisotropy of HAp is enhanced by the quantum Zeno effect.

The global decoherence of HAp under $\mathcal{H}_{D Q}$ was compared with that in adamantane, a regular 3D system, whose genuine many-body dynamics is manifested by the rapid excitation of very high orders of coherence. The coherence decays in both systems follow completely different functional forms.

In summary, we have addressed two main points:

(1) We confirmed the mapping of a nearest-neighbor onedimensional spin system under a double-quantum interaction to a noninteracting fermion system. This mapping was tested through one of its main consequences: the nonexcitability of 4-QC under $\mathcal{H}_{D Q}$. 
(2) We evaluated the decoherence through a Loschmidt echo experiment based on a double-quantum Hamiltonian. The restricted dynamics induced by the low connectivity space leads to the appearance of a smooth exponential decoherence, while the dynamics in a high connectivity space shows a sudden drop in coherence.

These results indicate that, in spite of residual interactions, HAp can be used as a "quantum simulator" for noninteracting fermion dynamics.

\section{ACKNOWLEDGMENTS}

The authors thank R. H. Acosta, Y. Garro Linck, and A. K. Chattah for fruitful experimental discussions, as well as L. E. F. Foa Torres and F. Pastawski for suggestions. This work was made possible through the financial support from CONICET, ANPCyT, and SeCyT-UNC. P.R.L. and H.M.P. acknowledge the hospitality of the Institute for Materials Science TU-Dresden and the MPI-PKS.
[1] J. R. Petta, A. C. Johnson, J. M. Taylor, E. A. Laird, A. Yacoby, M. D. Lukin, C. M. Marcus, M. P. Hanson, and A. C. Gossard, Science 309, 2180 (2005).

[2] M. V. G. Dutt, L. Childress, J. Liang, E. Togan, J. Maze, F. Jelezko, A. S. Zibrov, P. R. Hemmer, and M. D. Lukin, Science 316, 1312 (2007).

[3] D. D. Awschalom and M. E. Flatté, Nat. Phys. 3, 153 (2007).

[4] D. P. DiVincenzo, Science 270, 255 (1995).

[5] K. C. Nowack, F. H. L. Koppens, Y. V. Nazarov, and L. M. K. Vandersypen, Science 318, 1430 (2007).

[6] W. H. Zurek, Rev. Mod. Phys. 75, 715 (2003).

[7] W. H. Zurek, F. M. Cucchietti, and J. P. Paz, Acta Phys. Pol. B 38, 1685 (2007).

[8] D. G. Cory, A. F. Fahmy, and T. F. Havel, Proc. Natl. Acad. Sci. U.S.A. 94, 1634 (1997).

[9] L. M. K. Vandersypen and I. L. Chuang, Rev. Mod. Phys. 76, 1037 (2005).

[10] R. A. Jalabert and H. M. Pastawski, Phys. Rev. Lett. 86, 2490 (2001).

[11] G. A. Álvarez, E. P. Danieli, P. R. Levstein, and H. M. Pastawski, J. Chem. Phys. 124, 194507 (2006).

[12] G. A. Álvarez, E. P. Danieli, P. R. Levstein, and H. M. Pastawski, Phys. Rev. Lett. 101, 120503 (2008).

[13] G. A. Álvarez, Ph.D. thesis, Universidad Nacional de Córdoba, 2007 (unpublished) e-print arXiv:0705.2350.

[14] H. M. Pastawski, G. Usaj, and P. R. Levstein, Chem. Phys. Lett. 261, 329 (1996).

[15] Z. L. Mádi, B. Brutsher, T. Schulte-Herbrüggen, R. Brüschweiler, and R. R. Ernst, Chem. Phys. Lett. 268, 300 (1997).

[16] S. Bose, Phys. Rev. Lett. 91, 207901 (2003).

[17] M. Christandl, N. Datta, A. Ekert, and A. J. Landahl, Phys. Rev. Lett. 92, 187902 (2004).

[18] A. Kay, Phys. Rev. Lett. 98, 010501 (2007).

[19] T. D. Ladd, J. R. Goldman, A. Dana, F. Yamaguchi, and Y. Yamamoto, e-print arXiv:quant-ph/0009122v4 (2001).

[20] P. Cappellaro, C. Ramanathan, and D. G. Cory, Phys. Rev. Lett. 99, 250506 (2007).

[21] P. Cappellaro, C. Ramanathan, and D. G. Cory, Phys. Rev. A 76, 032317 (2007).

[22] H. M. Pastawski, P. R. Levstein, G. Usaj, J. Raya, and J. Hirschinger, Physica A 283, 166 (2000).

[23] P. R. Levstein, G. Usaj, and H. M. Pastawski, J. Chem. Phys. 108, 2718 (1998).

[24] H. G. Krojanski and D. Suter, Phys. Rev. A 74, 062319 (2006).

[25] J. Baum, M. Munowitz, A. N. Garroway, and A. Pines, J.
Chem. Phys. 83, 2015 (1985).

[26] R. R. Ernst, G. Bodenhausen, and A. Wokaun, Principles of Nuclear Magnetic Resonance in One and Two Dimensions (Oxford University Press, Oxford, 1987).

[27] S. I. Doronin, I. I. Maksimov, and E. B. Fel'dman, Zh. Eksp. Teor. Fiz. 118, 687 (2000).

[28] G. Cho and J. P. Yesinowski, Chem. Phys. Lett. 205, 1 (1993).

[29] G. Cho and J. P. Yesinowski, J. Phys. Chem. 100, 15716 (1996).

[30] C. P. Slichter, Principles of Magnetic Resonance (SpringerVerlag, New York, 1992).

[31] C. A. Pérez-Delgado, M. Mosca, P. Cappellaro, and D. G. Cory, Phys. Rev. Lett. 97, 100501 (2006).

[32] E. H. Lieb, T. Schultz, and D. C. Mattis, Ann. Phys. (N.Y.) 16, 407 (1961).

[33] E. B. Fel'dman and M. Rudavets, Chem. Phys. Lett. 311, 453 (1999).

[34] E. P. Danieli, H. M. Pastawski, and P. R. Levstein, Chem. Phys. Lett. 384, 306 (2004).

[35] S. I. Doronin and E. B. Fel'dman, Solid State Nucl. Magn. Reson. 28, 111 (2005).

[36] E. Rufeil-Fiori and H. M. Pastawski, Chem. Phys. Lett. 420, 35 (2006).

[37] B. Misra and E. C. G. Sudarshan, J. Math. Phys. 18, 756 (1977).

[38] P. R. Levstein, H. M. Pastawski, and R. Calvo, J. Phys.: Condens. Matter 3, 1877 (1991).

[39] H. M. Pastawski and G. Usaj, Phys. Rev. B 57, 5017 (1998).

[40] P. Facchi and S. Pascazio, Phys. Rev. Lett. 89, 080401 (2002).

[41] M. A. Voda, D. E. Demco, J. Perlo, R. A. Orza, and B. Blümich, J. Magn. Reson. 172, 98 (2005).

[42] R. H. Acosta, G. A. Monti, D. A. Vega, M. A. Villar, and E. M. Vallés, Macromolecules 39, 4788 (2006).

[43] M. Munowitz and A. Pines, Adv. Chem. Phys. 66, 1 (1986).

[44] H. Cho, T. D. Ladd, J. Baugh, D. G. Cory, and C. Ramanathan, Phys. Rev. B 72, 054427 (2005).

[45] J. Baum and A. Pines, J. Am. Chem. Soc. 108, 7447 (1986).

[46] Q. Zhang, Y. Leng, and R. Xin, Biomaterials 26, 2857 (2005).

[47] H. M. Pastawski, P. R. Levstein, and G. Usaj, Phys. Rev. Lett. 75, 4310 (1995).

[48] H. G. Krojanski and D. Suter, Phys. Rev. Lett. 93, 090501 (2004).

[49] C. M. Sánchez, H. M. Pastawski, and P. R. Levstein, Physica B 398, 472 (2007).

[50] M. Munowitz, Coherence and NMR (Wiley, New York, 1988).

[51] C. M. Sánchez, P. R. Levstein, R. H. Acosta, and A. K. Chattah (unpublished). 
[52] G. Manfredi and P.-A. Hervieux, Phys. Rev. Lett. 97, 190404 (2006).

[53] G. Manfredi and P.-A. Hervieux, Phys. Rev. Lett. 100, 050405 (2008).

[54] F. M. Cucchietti, H. M. Pastawski, and R. A. Jalabert, Phys.
Rev. B 70, 035311 (2004).

[55] T. Gorin, T. Prosen, T. Seligman, and M. Znidaric, Phys. Rep. 435, 33 (2006).

[56] P. Jacquod and C. Petitjean, Adv. Phys. (to be published) e-print arXiv:0806.0987v1. 